Eur. J. Clin. Chem. Clin. Biochem.

Vol. 32, 1994, pp. 529-537

(c) 1994 Walter de Gruyter \& Co.

Berlin · New York

\title{
Improvements in Creatinine Methodology:
}

\section{A Critical Assessment}

\author{
By B. G. Blijenberg, H. J. Brouwer, T. J. Kuller; R. Leeneman and C. J. M. van Leeuwen \\ University Hospital Rotterdam-Dijkzigt, Department of Clinical Chemistry, Rotterdam, The Netherlands
}

(Received March 18, 1994)

Summary: A large evaluation study, analytical as well as clinical, was performed on four published improvements for the determination of creatinine in serum. Two of the methods were based on the Jaffe reaction, the other two were enzymatic methods.

Analytically, all four methods showed a similar performance. In the analysis of numerous specimens from advanced care departments, however, the methods performed differently. The two enzymatic procedures scored better than the two Jaffé methods, when compared with an HPLC-based reference method. However, even the best methods produced outliers and a larger scatter than that obtained with "normal" specimens.

\section{Introduction}

In 1990 we initiated a study on the determination of creatinine in serum because we questioned the overall accuracy of our routine method (1). This study resulted in cooperation with both the Bayer and the DuPont company. Improvements in their Jaffé methods were published $(2,3)$.

Later, we studied four commercial methods based on an enzymatic reaction scheme. Special attention was paid to interference by bilirubin (4).

We now raise the question as to whether the current creatinine determination is satisfactory. All these earlier studies, done separately, revealed one superior method, although we always found outliers. Our daily routine creatinine is run on a Bayer-Technicon Chèm-1 (two instruments) while our emergencies are analysed with a DuPont Dimension.

Both methods compare well, but we still encounter problems, especially with very ill patients that need advanced care.

Therefore we decided to initiate a study with a large number of samples from our transplantation unit and general surgery intensive care unit, including many icteric specimens. We used the Chem-1 and the Dimen- sion, with the improved Jaffé methods, the best enzymatic method in our study, and a new enzymatic method that was described recently (5).

The rationale was the fact that both the Chem-1 and the Dimension are capable of operating open channel methods.

\section{Materials and Methods}

Materials

The raw materials were the same as described earlier (4).

Test Point unassayed chemistry control serum level 1 and 2 were from Technicon (Tarrytown, USA), and SRM 909a and $a_{2}$ Human Serum were from the National Institute of Standards and Technology (Gaithersburg, USA).

For spiking of the albumin solutions see Reference 4.

\section{Methods}

The current Chem-1 (called Chem-1, current) and Dimension (called Dimension, DuPont) creatinine methods were applied as described by the manufacturers.

The Boehringer enzymatic creatinine method (called Dimension, Boehringer) was modified as described in our previous publication (BM 3 4).

The Bayer enzymatic creatinine method (called Dimension, Bayer) was introduced as a Dimension open channel procedure according to the following assay conditions: 


\section{Sample}

Time of addition (s)

Volume $(\mu \mathrm{l})$

Wash $(\mu \mathrm{l})$

Mix

Reagent I

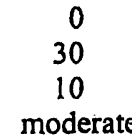

Time of addition (s)

Volume $(\mu \mathrm{l})$

Wash $(\mu l)$

Mix

Reagent 2

Time of addition (s)

Volume $(\mu \mathrm{l})$

Wash $(\mu \mathrm{l})$

Mix

$-96$

300

0

none

180
30
20
moderate

\section{Photometer}

Wavelength (nm)

340

700

210

Time of measurement 1 (s)

Time of measurement 2 (s)
The chemistry of this method has been described by Fossati et al (5). The reagents, Sera-Pak creatinine UV Method No. 6592, were provided by, Bayer and are not yet available for commercial use.

As a reference method we used our HPLC procedure (6).

\section{Patient samples}

Serum specimens used in this study were collected in various departments in the University Hospital Rotterdam. All samples were stored at $-70^{\circ} \mathrm{C}$ prior to use.

The icteric samples showed a total bilirubin concentration ranging from 79 to $886 \mu \mathrm{mol} / \mathrm{l}$. The transplantation sera came from patients after kidney, heart or liver transplantation. Samples from our surgery intensive care unit were not selected according to any criterion, except intensive care.

\section{Statistical analysis}

Regression analysis was done according to Passing \& Bablok (7).

Between-run variation was calculated using Test Point unassayed chemistry control serum level 1 ( $\pm 93 \mu \mathrm{mol} / 1$ in our daily routine) and level 2 ( $\pm 665 \mu \mathrm{mol} / \mathrm{l}$ in our routine) and both SRM samples (concentrations 84 and $463 \mu \mathrm{mol} / 1$ respectively).

\section{Instrumentation}

Both the Chem-1 and Dimension were used according to the manufacturer's instructions. For the calibration we followed the Technicon procedure for the Chem-1. The current Dimension was correlated precisely with the Chem-1, using patient samples.

Both enzymatic procedures were calibrated with the SRM 909 samples.

All methods were checked for accuracy during all runs with the same SRM 909 samples.

The recovery study was designed as a commutability study with samples used in the quality control schemes of the Dutch Quality Assessment Foundation (SKZL).

\section{Results}

The study consisted of an analytical part and a clinical evaluation. Most of the time our HPLC-based compari- son method was used as a reference method. All samples were analysed in duplicate.

\section{Analytical performance,}

The performance was studied by measuring imprecision, linearity, recovery of 30 different commercial samples and the interference of bilirubin-enriched artificial samples.

\section{Imprecision}

As mentioned in Materials and Methods, all results were obtained by using the SRM 909a values as calibrator points. Every run included both levels. The results measured were also used for calculation of the between-run coefficient of variation. In table 1 these data are given. We found comparable results for the bovine control samples (Test Point unassayed samples).

\section{Linearity}

We applied two approaches for the measurement of the linearity of patient samples and albumin standards. For the patient samples, the highest value used was 1329 $\mu \mathrm{mol} / 1$ (HPLC value) while the albumin standards ranged up to $1985 \mu \mathrm{mol} / \mathrm{l}$ (HPLC value).

In table 2 all information is tabulated.

Only the Bayer method declined slightly at levels higher than $1300 \mu \mathrm{mol} / \mathrm{l}$. This was confirmed with the albumin standards, and the highest concentration $(1985 \mu \mathrm{mol} / \mathrm{l})$ resulted in a value of $1929 \mu \mathrm{mol} / \mathrm{l}$.

Tab. 1 Imprecision methods.

Between-run variation for samples SRM $909 a_{1}$ and $a_{2}$ (stated valiues 84 and $463 \mu \mathrm{mol} / \mathrm{l}$ ).

\begin{tabular}{|c|c|c|c|c|}
\hline \multirow[t]{2}{*}{ Method } & \multicolumn{2}{|l|}{ SRM 909a } & \multicolumn{2}{|c|}{ SRM $909 a_{2}^{\prime}$} \\
\hline & $\begin{array}{l}\text { Creatinine } \\
(\mu \mathrm{mol} / \mathrm{l})\end{array}$ & $\begin{array}{l}\text { CV } \\
(\%)\end{array}$ & $\begin{array}{l}\text { Creatininine } \\
(\mu \mathrm{mol} / \mathrm{l})\end{array}$ & $\begin{array}{l}\text { CV } \\
(\%)\end{array}$ \\
\hline $\begin{array}{l}\text { Chem-1, current } \\
\quad(n=9)\end{array}$ & 81.6 & 6.2 & 469 & 2.9 \\
\hline $\begin{array}{l}\text { Dimension, DuPont } \\
\quad(n=9)\end{array}$ & 81.6 & 4.5 & 465 & 2.2 \\
\hline $\begin{array}{l}\text { Dimension, Boehringer } \\
\quad(n=9)\end{array}$ & 84.7 & 4.5 & 462 & 2.4 \\
\hline $\begin{array}{l}\text { Dimension, Bayer. } \\
\quad(n=5)\end{array}$ & 88.7 & 1.5 & 460 & 0.7 \\
\hline
\end{tabular}


Tab. 2 Patient samples (50) ranging 28-1329 $\mu \mathrm{mol} / \mathrm{l}$. Albumin standards (10) ranging 24-1985 $\mu \mathrm{mol} /$.

\begin{tabular}{lll}
\hline Method & \multicolumn{2}{l}{ Regression equations } \\
\cline { 2 - 3 } & $\begin{array}{ll}\text { Patient } \\
\text { samples }\end{array}$ & $\begin{array}{l}\text { Albumin } \\
\text { standards }\end{array}$ \\
\hline Chem-1, current & $y=1.10 x-2$ & $y=1.06 x-23$ \\
Dimension, DuPont & $y=1.02 x-1$ & $y=0.96 x-16$ \\
Dimension, Boehringer & $y=1.01 x+3$ & $y=1.06 x-10$ \\
Dimension, Bayer & $y=0.98 x+7$ & $y=0.98 x+7$ \\
\hline
\end{tabular}

$x=$ HPLC-method

$y=$ method under study

\section{Recovery}

We only used those commercial samples with creatinine values lower than $300 \mu \mathrm{mol} / \mathrm{l}$. All the results are shown in figure 1 .

\section{Bilirubin interference}

Interference by conjugated and unconjugated bilirubin in a human albumin based creatinine standard was investigated for all the methods under study. The results are shown in figures 2 and 3.

Total bilirubin concentrations were measured with the Chem-1 method according to the instructions of the manufacturer.

\section{Clinical evaluation}

In the clinical part of the study we analysed 388 samples from 312 patients. There were roughly equal numbers of samples from icteric patients, transplanted patients and intensive care patients.

It was possible to trace the samples, if more clinical information was required.

In the graphs we restricted ourselves to creatinine values lower than $300 \mu \mathrm{mol} / \mathrm{l}$. All methods were studied with roughly the same number of equal samples, with the exception of the Bayer method, the study of which was started at a later stage. At the time, it was most convenient for us to use only the Dimension as the analysing instrument. Later, we will also evaluate the Chem-1, as we are in the process of adapting the Bayer method to this instrument.

\section{Samples from icteric patients}

To present a single impression of an $x, y$-diagram for each of the various method comparisons, all results of the determinations with the icteric specimens are depicted in figure 4.
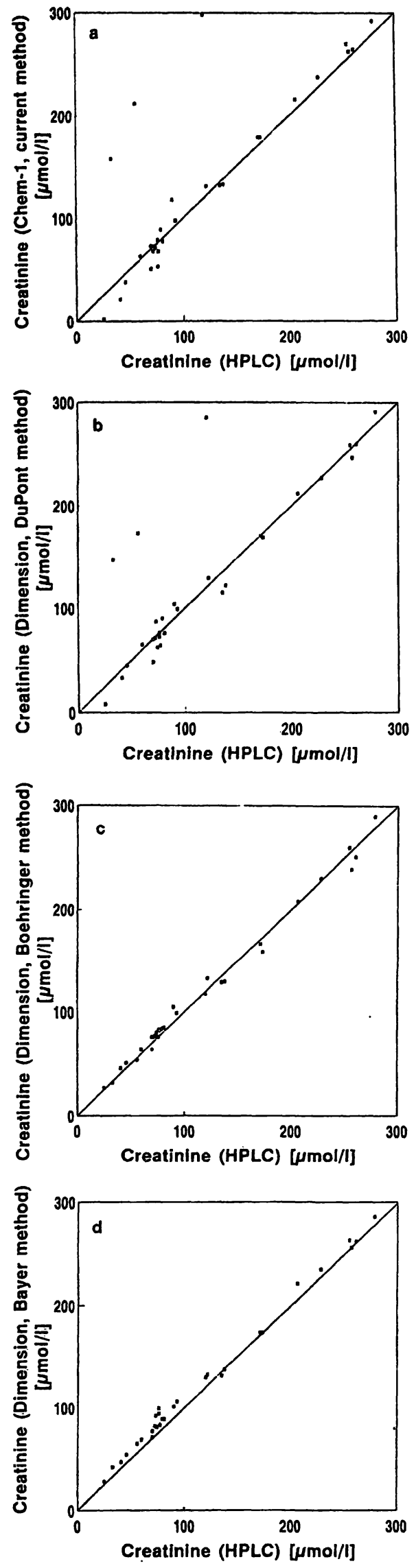

Fig. 1 Comparison of methods under study (y-axis) and our HPLC based reference method ( $x$-axis) using 30 different commercial control sera:
a) Chem-1, current method
b) Dimension, DuPont method
c) Dimension, Boehringer method
d) Dimension, Bayer method 
More detailed information can be obtained from figures 5 , where we plotted the residuals versus the creatinine levels, and from figure 6 , where we did the same versus the bilirubin concentrations.

\section{Samples from transplanted patients}

Here, only the residuals versus the creatinine concentrations are plotted (see fig. 7).

\section{Samples from intensive care patients}

The same choice was made for these samples, i. e. only the results of the residuals are plotted (see fig. 8).

\section{Discussion}

As we stated in the Introduction, much effort has been spent in evaluating a number of modifications of the

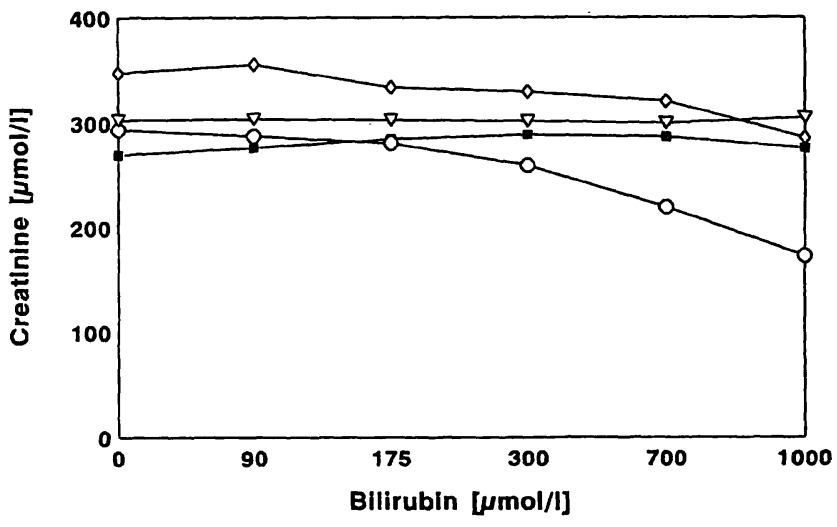

Fig. 2 The influence of unconjugated bilirubin (added as bilirubin-enriched albumin solutions) on methods under study:

$\diamond=$ Chem- 1 , current method

$\mathbf{a}=$ Dimension, DuPont method

$O=$ Dimension, Boehringer method

$\nabla=$ Dimension, Bayer method

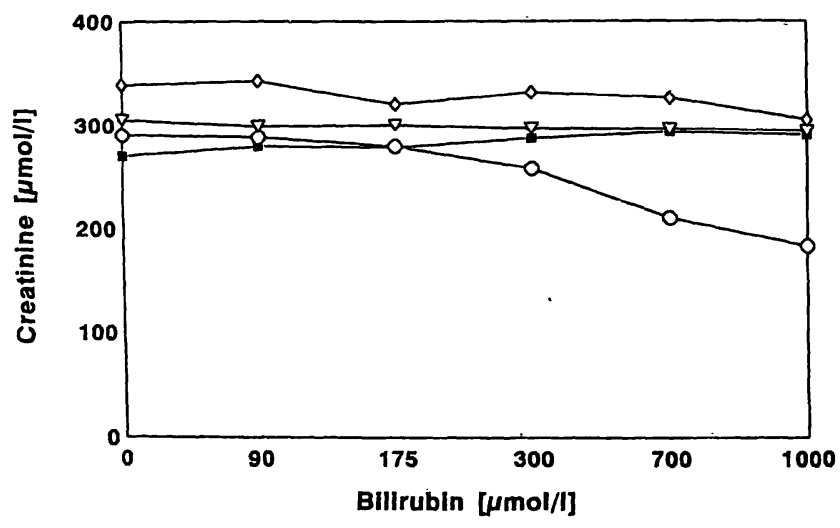

Fig. 3 The influence of ditaurobilirubin (added as ditaurobilirubin-enriched albumin solutions) on methods under study:

$\diamond=$ Chem-1, current method

a = Dimension, DuPont method

$O=$ Dimension, Boehringer method

$\nabla=$ Dimension, Bayer method
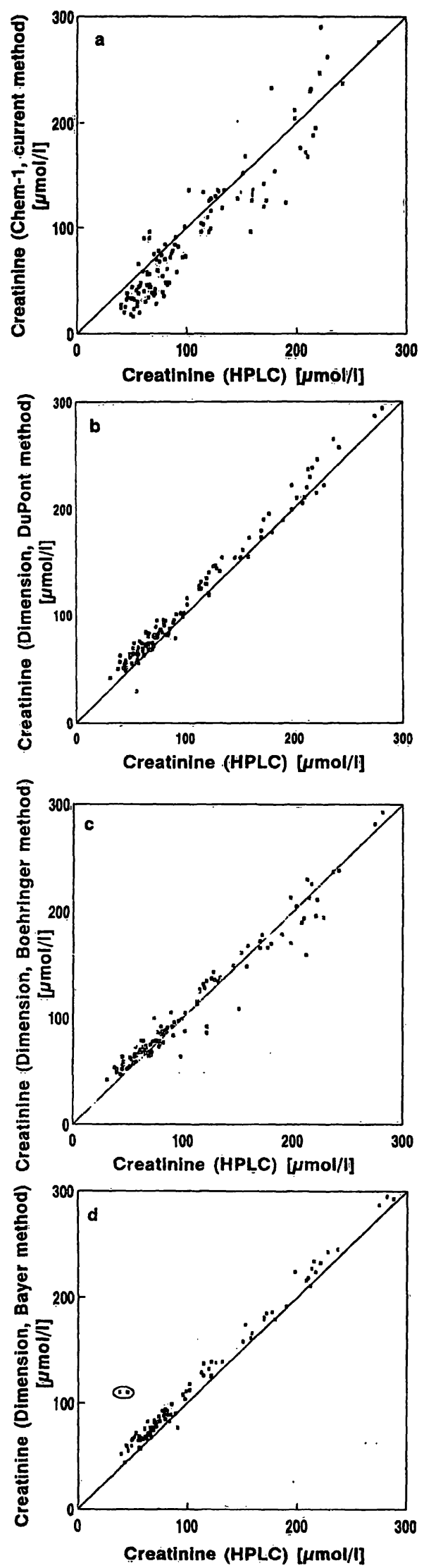

Fig. 4 Comparison of methods under study (y-axis) and our HPLC based reference method ( $x$-axis) using icteric patient samples:

a) Chem-1, current method

b) Dimension, DuPont method

c) Dimension, Boehringer method

d) Dimension, Bayer method The circled points refer to samples containing 5-fluorouracil (see
Discussion). 

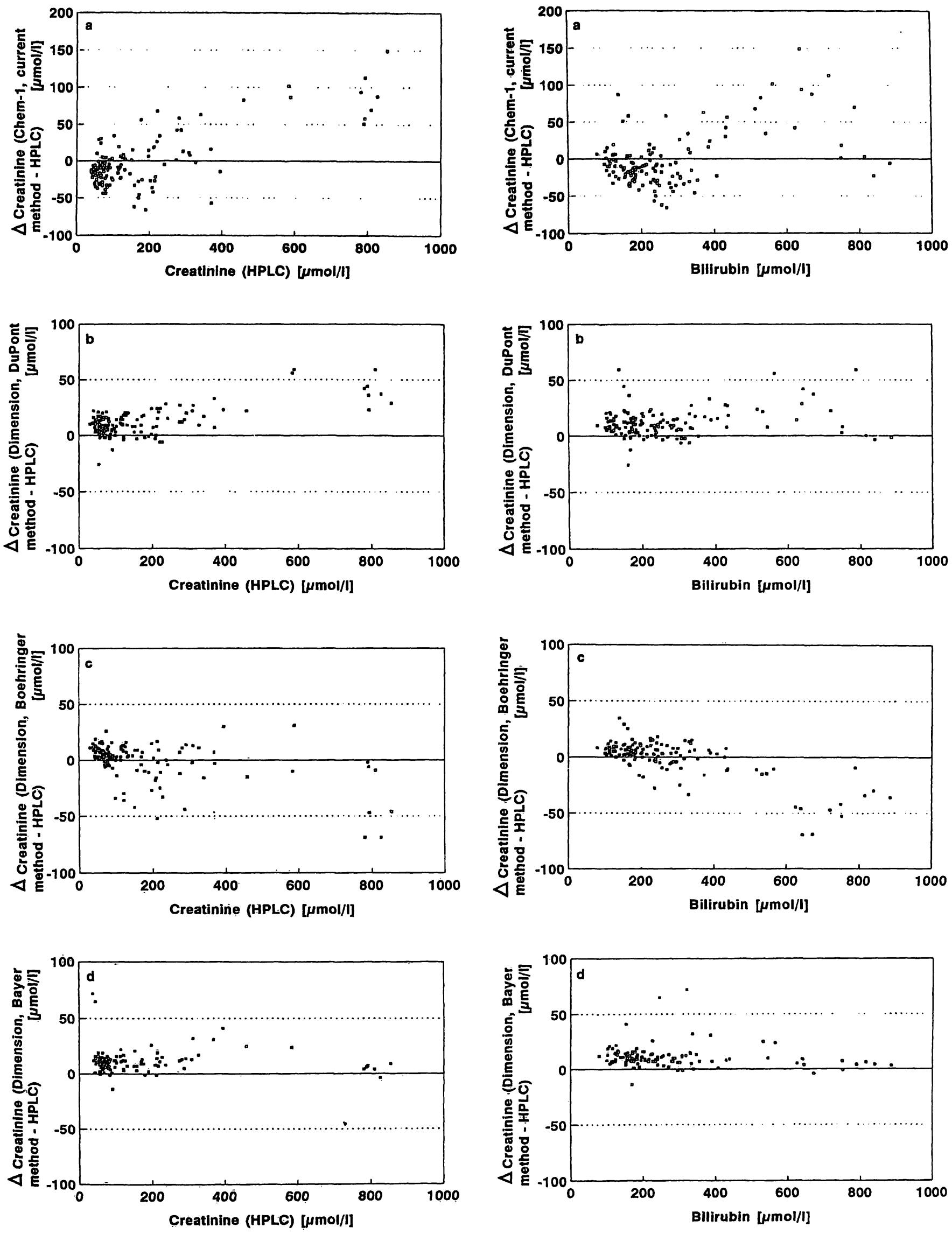

Fig. 5 Residual creatinine values of icteric sera plotted against the creatinine (HPLC) concentration for the methods under study:

a) Chem-1, current method

b) Dimension, DuPont method

c) Dimension, Boehringer method

d) Dimension, Bayer method

Fig. 6 Residual creatinine values of icteric sera plotted against the bilirubin concentration for the methods under study:
a) Chem-1, current method
b) Dimension, DuPont method
c) Dimension, Boehringer method
d) Dimension, Bayer method

Eur. J. Clin. Chem. Clin. Biochem. / Vol. 32, 1994 / No. 7 

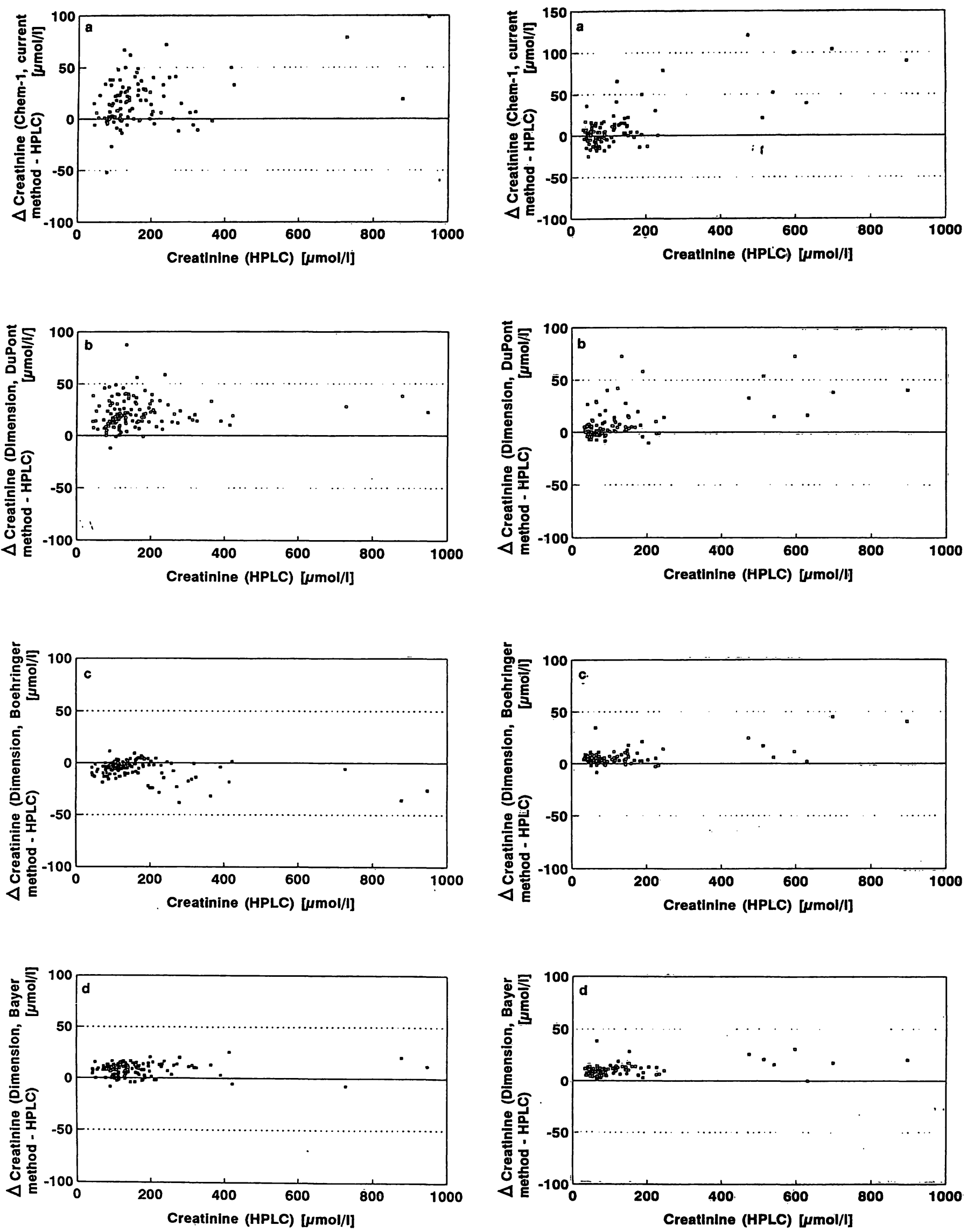

Fig. 7 Residual creatinine values of transplanted patients' samples plotted against the creatinine (HPLC) concentration for the methods under study:
a) Chem-1, current method
b) Dimension, DuPont method
c) Dimension, Boehringer method
d) Dimension, Bayer method

Fig. 8 Residual çreatinine vạlues of intensive care patients' sam= ples plotted against the creatinine (HPLC) concentration for the methods under study:

a) Chem-1, current method

b) Dimension, DuPont method

c) Dimension, Boehringer method

d) Dimension, Bayer method 
determination of creatinine in serum. Although the creatinine determination in clinical practice is nearly 100 years old (8), there still is much debate regarding its accuracy (9). Numerous articles have been written on modifications and improvements of the various reaction principles, be it the Jaffé reaction or an enzymatic procedure.

We have also added to this long list of publications, for the same reason: dissatisfaction with the existing procedures. We cooperated with some manufacturers on their method modifications, and we were able to publish improvements, without being sure that we had achieved absolute accuracy. The same held true for our own modifications of some enzymatic methods.

Knowing that we had now reached the state-of-the-art with the above-mentioned manufacturers, we wanted to make a choice for ourselves. Therefore we organized a large study, which was analytical as well as clinical.

As mentioned earlier, we use two Chem-1 systems in our routine section, and a Dimension for our emergencies. Correlation of the creatinine determinations on these instruments occupies much of our attention, in view of the plausibility study of our quality control scheme. The numerous drugs, in particular to the many advanced care patients in our large hospital, sometimes give rise to significant differences in the results obtained with the Chem-1 and the Dimension, despite the perfect correlation normally seen.

In order to complete the statistical evaluation, all regression characteristics are given in table 3 .
Considering all information in the figures and mentioned in table 3, we feel that both enzymatic procedures score better than both Jaffé methods. The performances of the two enzymatic methods are more or less comparable, while the two Jaffé methods show certain differences, the Chem-1 modification generally showing a worse performance. It is not possible to compare our Boehringer modification and the Bayer method completely, because the number of samples differ.

At the time of inclusion of the Bayer method, the number of icteric samples was becoming depleted (98 versus 123). It is just this section where the Boehringer method scatters more than the Bayer method (fig. $5 \mathrm{c}$ and 5d). Here the Bayer method graph shows two interesting results, which were traced to one patient with metastasized breast carcinoma, receiving a number of drugs at the time the samples were taken. These drugs included 5-fluorouracil which has the same type of molecular structure as 5-fluorocytosine, which was listed by Fossati et al. (5) as an in vitro interferent.

With respect to bilirubin interference, in vitro and in vivo (figs. 2, 3, 6c and 6d), the Bayer method scores better in the higher bilirubin range $(>400 \mu \mathrm{mol} / \mathrm{l})$. On the other hand, the linearity study suggests that the Boehringer method may be somewhat better than the Bayer method. Fossati et al. found a better linearity (up till $1768 \mu \mathrm{mol} / \mathrm{l})$. In the daily clinical situation, however, the linearity of both methods is quite acceptable.

Both the current Chem-1 and the Dimension methods are based on the Jaffé reaction. There is one striking

Tab. 3 Statistical evaluation.

Range of creatinine concentrations: $0-1000 \mu \mathrm{mol} / \mathrm{l}$.

\begin{tabular}{lll}
\hline Samples & Methods & Regression \\
\hline "Normal" & Chem-1, current & $\mathrm{y}=1.11 \mathrm{x}-3 ; \mathrm{r}=1.00 ; \mathrm{n}=46$ \\
Icteric & Chem-1, current & $\mathrm{y}=1.15 \mathrm{x}-28 ; \mathrm{r}=0.99 ; \mathrm{n}=132$ \\
Transplantation & Chem-1, current & $\mathrm{y}=1.07 \mathrm{x}+4 ; \mathrm{r}=0.99 ; \mathrm{n}=104$ \\
Intensive care & Chem-1, current & $\mathrm{y}=1.14 \mathrm{x}-12 ; \mathrm{r}=0.99 ; \mathrm{n}=98$ \\
"Normal" & Dimension, DuPont & $\mathrm{y}=1.03 \mathrm{x}-1 ; \mathrm{r}=1.00 ; \mathrm{n}=46$ \\
Icteric & Dimension, DuPont & $\mathrm{y}=1.05 \mathrm{x}+4 ; \mathrm{r}=1.00 ; \mathrm{n}=140$ \\
Transplantation & Dimension, DuPont & $\mathrm{y}=1.02 \mathrm{x}+19 ; \mathrm{r}=1.00 ; \mathrm{n}=130$ \\
Intensive care & Dimension, DuPont & $\mathrm{y}=1.07 \mathrm{x}-1 ; \mathrm{r}=0.97 ; \mathrm{n}=92$ \\
"Normal" & Dimension, Boehringer & $\mathrm{y}=1.03 \mathrm{x}-1 ; \mathrm{r}=1.00 ; \mathrm{n}=46$ \\
Icteric & Dimension, Boehringer & $\mathrm{y}=0.96 \mathrm{x}+7 ; \mathrm{r}=1.00 ; \mathrm{n}=140$ \\
Transplantation & Dimension, Boehringer & $\mathrm{y}=1.03 \mathrm{x}+2 ; \mathrm{r}=1.00 ; \mathrm{n}=130$ \\
Intensive care & Dimension, Boehringer & $\mathrm{y}=1.05 \mathrm{x} ; \mathrm{r}=1.00 ; \mathrm{n}=100$ \\
"Normal" & Dimension, Bayer & $\mathrm{y}=0.98 \mathrm{x}+8 ; \mathrm{r}=1.00 ; \mathrm{n}=46$ \\
Icteric & Dimension, Bayer & $\mathrm{y}=1.00 \mathrm{x}+11 ; \mathrm{r}=1.00 ; \mathrm{n}=111$ \\
Transplantation & Dimension, Bayer & $\mathrm{y}=1.01 \mathrm{x}+7 ; \mathrm{r}=1.00 ; \mathrm{n}=128$ \\
Intensive care & Dimension, Bayer & $\mathrm{y}=1.02 \mathrm{x}+8 ; \mathrm{r}=1.00 ; \mathrm{n}=100$ \\
\hline
\end{tabular}

$\begin{array}{ll}\mathbf{x} & =\text { HPLC-method } \\ \mathbf{y} & =\text { method under study } \\ \text { "Normal" } & =\text { non-icteric, non-haemolytic and non-lipaemic }\end{array}$

Eur. J. Clin. Chem. Clin. Biochem. / Vol. 32, 1994 / No. 7 
difference regarding the correction for the bilirubin interference. The Chem-1 uses a selective bichromatic measurement for icteric samples while in the Dimension formulation bilirubin is oxidised by potassium hexacyanoferrate(III). The in vitro experiments with bilirubinenriched samples show an acceptable performance for both methods (figs. 2 and 3 ) while the icteric patient samples give a larger scatter, especially with the Chem1 (figs. 4a, 4b, 5a and 5b).

This may mean that oxidation of bilirubin is a better way of circumventing the interference than the use of a mathematical algorithm. We have no experience of, and are not aware of any detailed literature on this question $(10,11)$. The experiments with specimens from transplanted patients confirm that the kinetics of the Jaffé reaction probably also contribute to the differences in the results of the two methods. We observed no abnormal colouring of the samples, which had been submitted for cyclosporin assays. However, it is certain that many more drugs $\$ were involved.

It may also be helpful to compare the results presented in the residual graphs for the pathological specimens (icteric, transplantation and intensive care) with those obtained with "normal" serum samples. Here, normal is defined as non-icteric, non-haemolytic and non-lipaemic. In figure 9 , only the data related to the Bayer method are given as an example of the observed scatter. The other methods show a comparable picture.

The variation with pathological sera is higher than with "normal" sera for all methods. Especially in the clinically most relevant range $(<300 \mu \mathrm{mol} / \mathrm{l})$, there is still

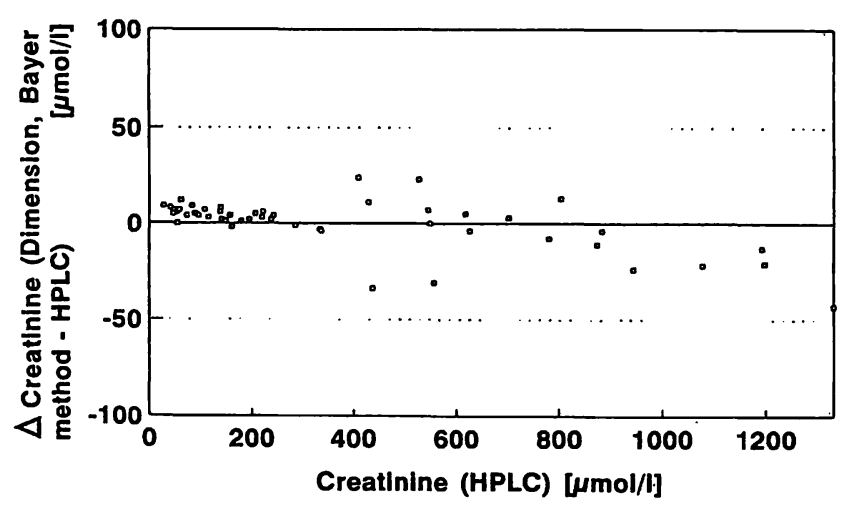

Fig. 9 Residual creatinine values of "normal" sera plotted against the creatinine (HPLC) concentration for the Dimension, Bayer method. room for an improvement of accuracy, although it is clear that the two enzymatic methods perform better than the Jaffé-based methods.

An overall picture of all residual graphs shows a calibration problem. All measurements were done within a few weeks and where possible grouped together according to the nature of the samples. Differences are particularly apparent in the Chem-l results: the average residual is negative for icteric specimens, positive for transplantation specimens and about zero for intensive care samples. The most positive picture is shown by another Bayer development, i. e. the Bayer enzymatic procedure.

An interesting situation is revealed in the graphs of the commercial samples (fig. 1), and this clearly reflects the problems we meet in assessing surveys. We had the same experience last year in the Netherlands and earlier in Germany. Thus, application of reference methodology (the correct way) may lead sometimes to difficulties in interpretation with particular tests. Here too, it has to be emphasized that both Jaffé methods perform less well than the enzymatic methods.

In the Introduction we raised the question: are we satisfied with the improvements? We are sure that we can improve our daily situation in theory. The least we can do, considering that both the Chem -1 and the Dimension can now be used as open systems, is to try to use a single method. From our ranking, it is clear that we have to replace the current Chem- 1 method. At the moment we have no practical experience to support this move, but this and the financial implications must be faced, especially regarding the introduction of enzymatic procedures. There is one practical limitation, i. e. the Bayer method is not yet commercialized.

Finally, we are not entirely negative regarding our current Chem- 1 method. We have been running this method for almost one year, and it performs well for the majority of specimens, as shown by our quality control scheme, as well as all studies we have performed. Nevertheless, it is clear that we can do better, and that is also our wish.

\section{Acknowledgement}

Many thanks are due to $L$. Zwang for his data handling support and to A. P. Copper-Staamer for her clerical assistance. Furthermore we want to thank $W$. Buijteweg (Miles, Tarrytown, USA) for his help in providing the Bayer method reagents.

\section{References}

1. Blijenberg, B. G., Liesting, E. C. \& Zwang, L. (1992) Creatinine and automatic analysers in relation to icteric specimens. Eur. J. Clin. Chem. Clin. Biochem. 30, 779-784.
2. Blijenberg, B. G., Zwang, L. \& Van Tilborg, M. (1993) New approaches with the Chem-1 creatinine determination. Eur. J. Clin. Chem. Clin. Biochem. 31; 29-33. 
3. Blijenberg, B. G., Zwang, L. \& Klein Heerenbrink, G. J (1993) Serum creatinine and the DuPont Dimension: Possible improvements. Eur. J. Clin. Chem. Clin. Biochem. 31, 147151.

4. Schoenmakers, C. H. H., Kuller, T., Lindemans, J. \& Blijenberg, B. G. (1993) Automated enzymatic methods for creatinine measurement with special attention to bilirubin interference. Eur. J. Clin. Chem. Clin. Biochem. 31, 861-866.

5. Fossati, P., Ponti, M., Passoni, G., Tarenghi, G., Melri d'Eril, G. v. \& Prencipe, L. (1994) A step forward in enzymatic measurement of creatinine. Clin. Chem. 40, 130-137.

6. Zwang, L. \& Blijenberg, B. G. (1992) Validation of the ultrafiltration technique for creatinine analysis by HPLC: A comparison with direct serum injection. Eur. J. Clin. Chem. Clin. Biochem. 30, 861-866.

7. Passing, H. \& Bablok, W. (1983) A new biomedical procedure for testing the equality of measurements from two different analytical methods. J. Clin. Chem. Clin. Biochem. 21, 709720.
8. Folin, O. (1904) Beitrag zur Chemie des Kreatinins und Kreatins im Harne. Z. Physiol. 41, 223.

9. Spencer, K. (1986) Analytical reviews in clinical biochemistry: The estimation of creatinine. Ann. Clin. Biochem. 23, 1-25.

10. Rajs, G. \& Mayer, M. (1992) Oxidation markedly reduces bilirubin interference in the Jaffé creatinine assay. Clin. Chem. $38,2411-2413$.

11. Knapp, M. L. \& Mayne, P. D. (1987) Development of an automated kinetic Jaffé method designed to minimise bilirubin interference in plasma creatinine assays. Clin. Chim. Acta 168 , $239-246$.

Dr. B. G. Blijenberg

University Hospital Rotterdam-Dijkzigt

Department of Clinical Chemistry

Dr. Molewaterplein 40

NL-3015 GD Rotterdam

The Netherlands 
